\title{
A Vehicle Seat Rattle Noise Diagnosis Study Case
}

\author{
Chunqing YANG ${ }^{1, a, ~}{ }^{,}$, Cansong $\mathrm{GU}^{2, \mathrm{~b}}$, Lili SU ${ }^{3, \mathrm{c}}$ \\ ${ }^{1}$ China Automotive Technology \& Research Center, Tianjin, 300300, China \\ ${ }^{2}$ China Automotive Technology \& Research Center, Tianjin, 300300, China \\ ${ }^{3}$ China Automotive Technology \& Research Center, Tianjin, 300300, China \\ ayangchunqing@catarc.ac.cn, bgucansong@catarc.ac.cn, csulili@catarc.ac.cn, ”corresponding \\ author
}

Keywords: BSR (Buzz Squeak and Rattle), Wavelet Transform, Relative Approach3D

\begin{abstract}
Vehicle seat rattle noise is one of the common issues in automotive. In general, BSR (Buzz Squeak and Rattle) noises are directly linked to the vehicle NVH (Noise Vibration and Harshness) performance. Most importantly, customers perceive BSR as direct indicator of vehicle build quality and durability. Diagnosing source of BSR is one critical step to solve the issue and it is still very challenging work due to the uncertainty of the impact mechanism at joints which contribute to the BSR. Generally, vehicle on road testing is used to detect the BSR and lab test is used to reconstruct the problems for further measurement and analysis. In this paper, a case of seat rattle noise study started from on road evaluation first, then the seat was installed on a vibration test bench and sound pressure and acceleration were measured in hemi anechoic lab environment. Multiple VNH testing systems, such as LMS Test Lab, Head Acoustic ArtemiS and MB Dynamics were used since each system has unique capability which is necessary for this study. MB Dynamics was used to reconstruct rattle noise condition in hemi anechoic lab environment. LMS Test Lab Wavelet transform and Head ArtemiS Relative Approch3D were adopted in measurement and analysis. Based on sound and vibration measurement, the rattle noise source was identified, confirmed and quantified. It was found that rattle noise originates from the joint of headrest assembly and seat back. The diagnosis results lead to a fairly easy solution to solve the rattle noise issue.
\end{abstract}

\section{Introduction}

Customers increasingly perceive BSR as direct indicator of vehicle quality and durability [1]. Generally, squeak is a friction induced noise from two solid surfaces in contact sliding in the opposite direction against each other. One of the fundamental squeak noise mechanisms is unstable vibration that has stick-slip motion characteristics. Frequencies of squeak noise usually range from $200 \mathrm{~Hz}$ to $8 \mathrm{kHz}$. Rattle noise is impact-induced noise and its low frequency vibration contact generates broad band frequency noise with the majority content at low frequency, its frequency range could be from $50 \mathrm{~Hz}$ to $8 \mathrm{kHz}$ [2-5]. Solving BSR issue is very challenging since it is transient or come-and-go style. Its presence is depending on the roads surface, vehicle speed and temperature, etc. Conducting BSR noise measurement on road sometime could be time consuming and fruitless on test track. Also, quite often, diffused vehicle interior sound field makes measurement of BSR more difficult due to the masking effect from other noise sources. Reconstructed rattle noise condition in lab environment is preferred and it enables engineers to repeat and measure in much 
quiet environment with a piece of easy mind. In this paper, a vehicle has interior rattle noise complaints was driving through different road surfaces. It was found out that the rattle noise presences every time when driving through one testing track called Smooth Belgian road at $40 \mathrm{~km} / \mathrm{h}$ speed. Subjective evaluation points to upper portion of the passenger seat assembly. However, it is difficult to pinpoint the rattle noise source. Therefore, Power Spectrum Density (PSD) of the road input when rattle noise is occurring on this track was recorded, it was used as input for reconstructed seat rattle noise in lab environment. The problematic seat was installed on vibration test bench, sound and vibration measurements were completed by Head Acoustics SQuadriga 11. LMS Test lab was used for Wavelet Transform analysis and Head ArtemiS was adopted for Relative Approch3D calculation.

\section{Rattle Noise On-road Evaluation}

A vehicle has interior rattle noise was received, it was reported that the rattle noise was only present on certain road condition and it is kind of come-and-go transient noise. This vehicle was driving at different speeds on different test tracks. The rattle noise was finally occurring when it was on Smooth Belgian Road testing track, showing in Figure1, at $40 \mathrm{~km} / \mathrm{h}$ speed. Subjective evaluation of this rattle noise points to the passenger seat, however interior sound pressure level (SPL) can only show nothing more than typical vehicle interior SPL spectrum, neither it can tell where on the seat assemble the rattle noise comes from, nor does it indicates clear peak frequencies due to the masking effect from other sources. In order to reconstruct the rattle noise in lab environment, one tri-axial accelerometer and one single-axial accelerometer were attached to the seat mounts. LMS test lab was used to record these accelerometer signals (Figure 2). These signals will be used as input source for MB Dynamics vibration testing bench when reconstructing seat rattle noise in lab.

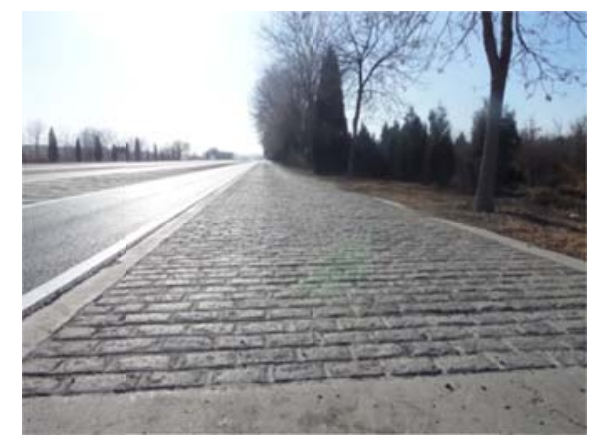

Figure 1. Smooth Belgian road

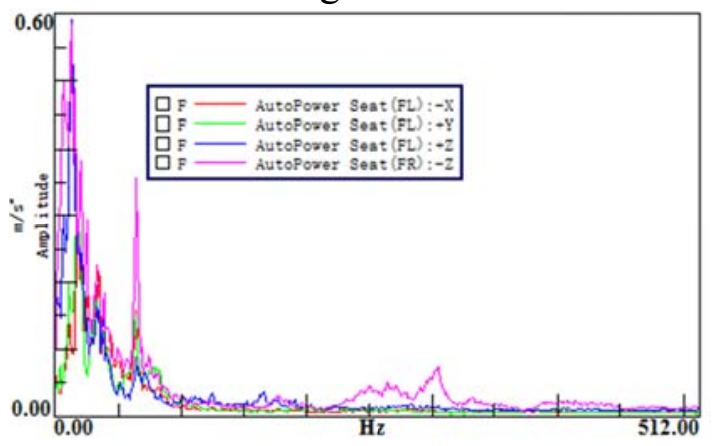

Figure 2. Acceleration signals of seat mountings

\section{Rattle Noise in Lab Measurement}

In hemi-anechoic lab environment, vibration testing bench is controlled by MB Dynamics (Figure 3), which are controlled by MB Dynamic Testing software. The PSD profiles recorded on Smooth Belgian Road at $40 \mathrm{~km} / \mathrm{h}$ speed are fed into the shakers to drive the test bench. 

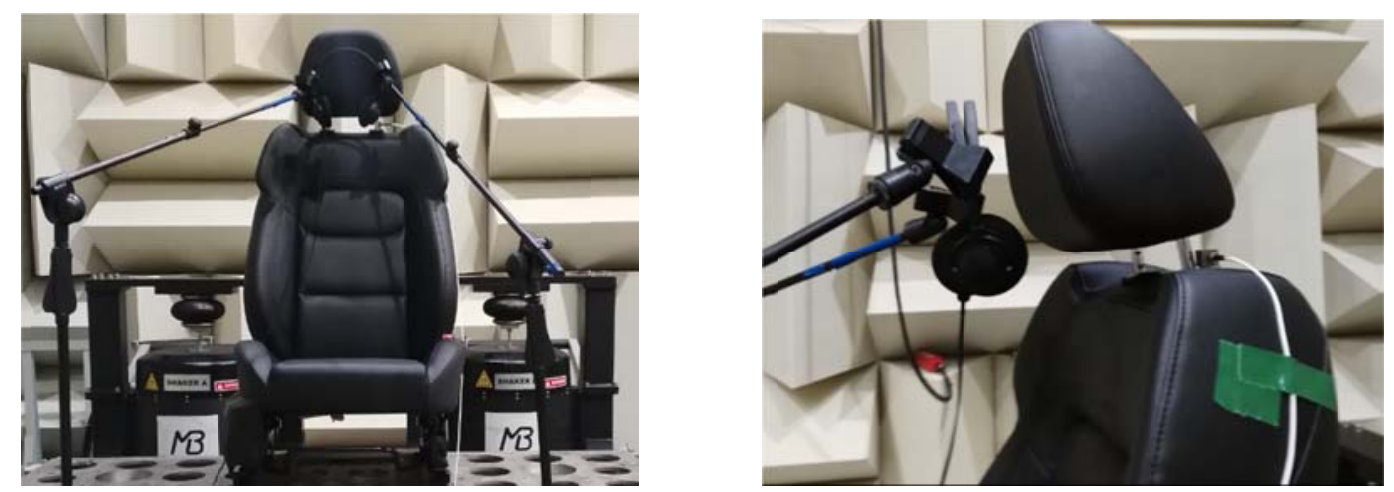

Figure 3. MB Dynamics shaker system and measuring position Power Spectral Density (PSD)

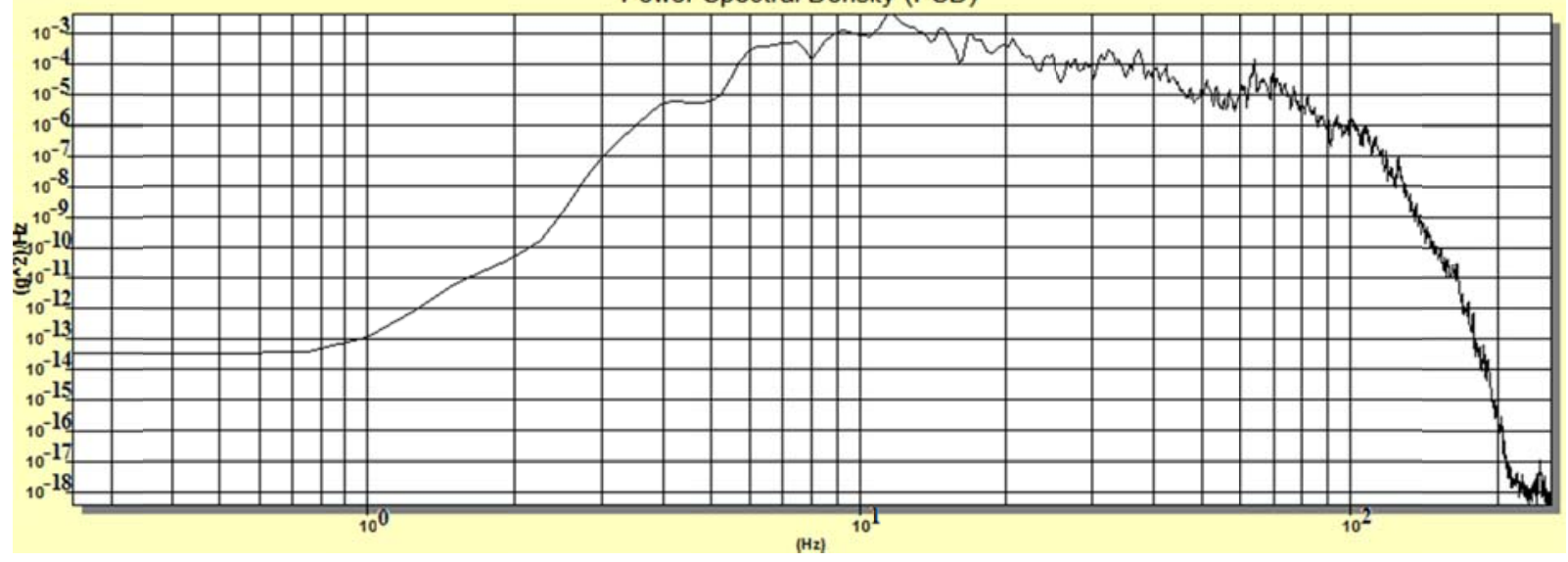

Figure 4. Seat PSD profile of MB Dynamics software

Figure 4 shows seat PSD profile which MB Dynamics used as controlling strategy. Headset microphone was placed at passenger head positon. Since subjective evaluation on road points to the upper portion of the seat, one tri-axial accelerometer was mounted to the headrest mounting guide. SPL and vibration acceleration level were measured by HEAD Acoustics SQuadriga 11. 10 seconds time history files of both sound pressure and acceleration signals were recorded.

\section{Wavelet Transform Analysis}

Wavelet Transform technology of LMS test lab was used for analyzing the SPL and the acceleration signal. Wavelet Transform is used broadly for capturing the transient phenomenon of abnormal noise. A wavelet is a mathematical function used to divide a given function or continuous-time signal into different scale components. The Wavelet Transform is often compared with the Fourier Transform, in which signals are represented as a sum of sinusoids. The main difference is that wavelets are localized in both time and frequency whereas the standard Fourier Transform is only localized in frequency. The short-time Fourier Transform is more similar to the Wavelet Transform, but there are issues with the frequency/time resolution trade-off. Wavelet often give a better signal representation using multi-resolution analysis, with balanced resolution at any time and frequencies [6].

The Wavelet Transform of SPL showing in figure 5 and figure 6 indicates there are some transient noises peak occur during the measurement at the condition seat with headrest. Figure 5 shows the unoccupied seat with headrest condition and figure 6 shows unoccupied seats without headrest condition. Multiple high amplitude spectrum lines (light green color strips) appear when the seat had headrest and nearly disappeared or being mitigated when headrest was removed. This indicates that the joint of headrest assembly and seat back assembly was the source for the rattle noise. 


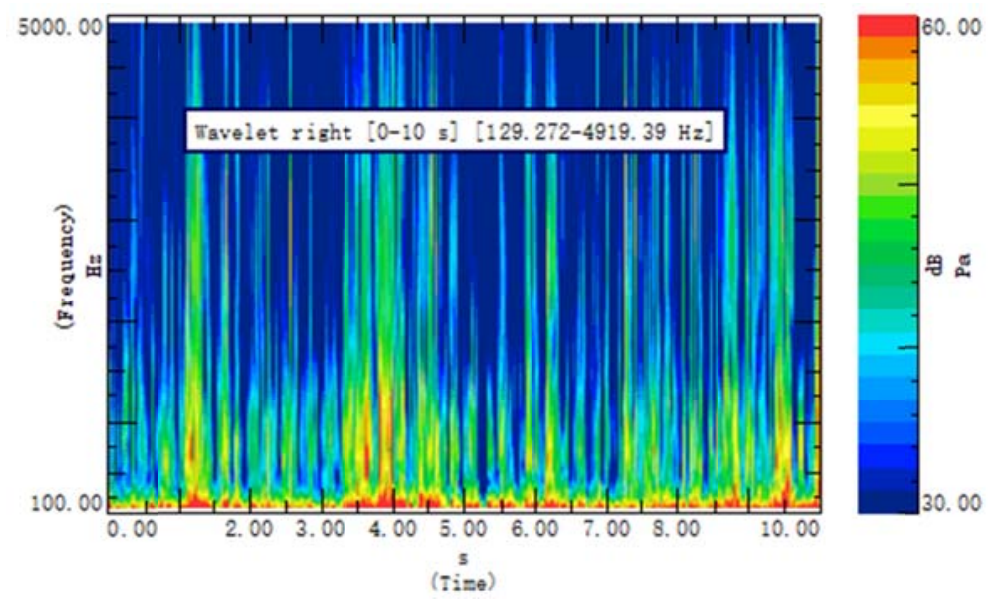

Figure 5. Sound pressure level Wavelet Transform of seat with headrest

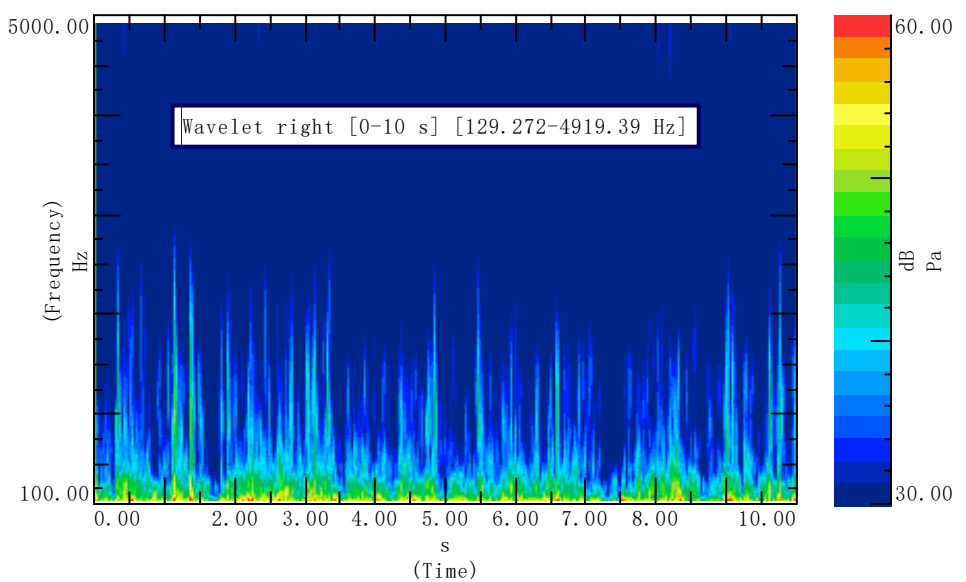

Figure 6. Sound pressure level Wavelet Transform of seat without headrest

Wavelet Transform analysis was also used to analyze the vibration. Figure 7 and Figure 8 show the acceleration vs frequency and time. At without headrest condition, the peak spectrum lines were significantly mitigated in Figure 8 compared with Figure 7 which the seat had headrest on. Vibration Wavelet Transform analysis again identifies that the rattle noise comes from this joint area.

After qualitative Wavelet Transform, Relative Approch3D is used to conduct quantitative analysis. The analysis mainly focuses on sensitive frequencies of human hearing.

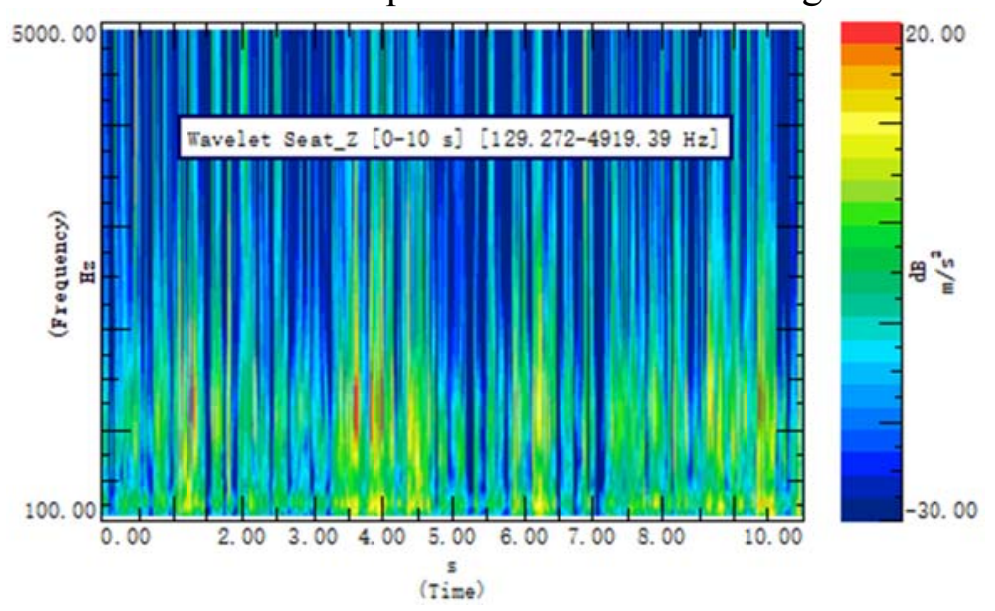

Figure 7. Vibration acceleration- Wavelet Transform of seat with headrest 


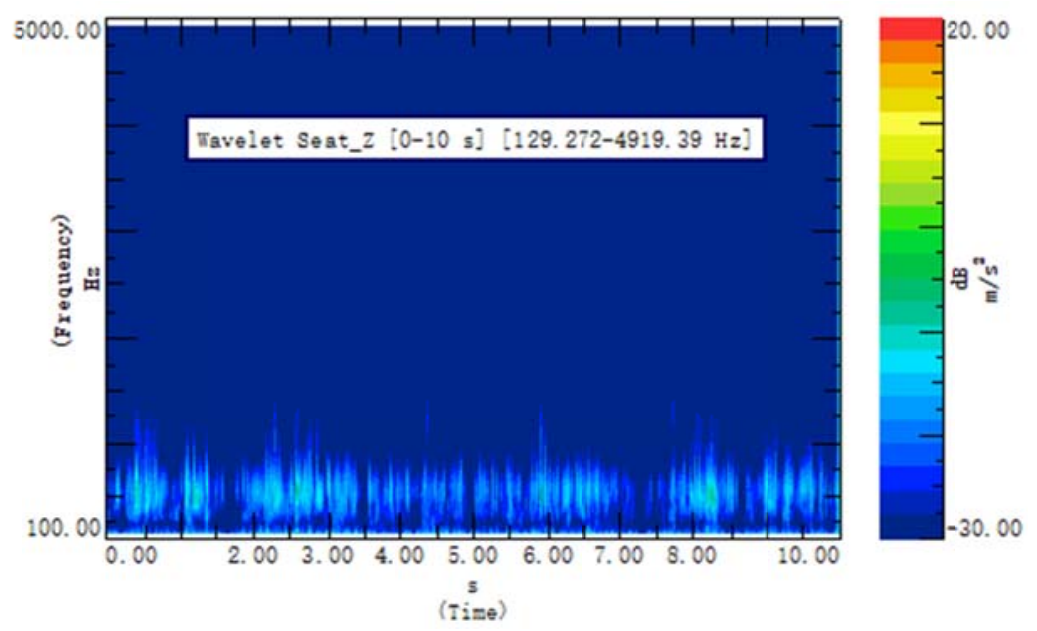

Figure 8. Vibration acceleration -Wavelet Transform of seat without headrest

Compared figure 5 with figure 7, it can be seen that SPL and vibration acceleration level has a very good correlation. This again indicates that the rattle noise comes from the joint area of the passenger seat headrest.

\section{Relative Approach3D}

Relative Approach3D calculation is another analysis technique from ArtemiS. Relative Approch3D indicates the auditory sense of the noise by visual sense of the graph. The Relative Approach algorithm looks for signal changes in time and frequency domain. It looks for the sensitive frequencies of human hearing. The frequency of rattle noise ranges from $400 \mathrm{~Hz}$ to $4 \mathrm{kHz}$ in this case. An estimate for the spectral data based on the previous $200 \mathrm{~ms}$ and both frequency directions is calculated. The difference of actual data and its estimate is the result of the relative approach. The $1 / \mathrm{n}$ Octave analysis can be implemented by time domain filters or FFT-based, the band resolution may be adjusted. Both the graphs have the band resolution of $24^{\text {th }}$ octave in this case.

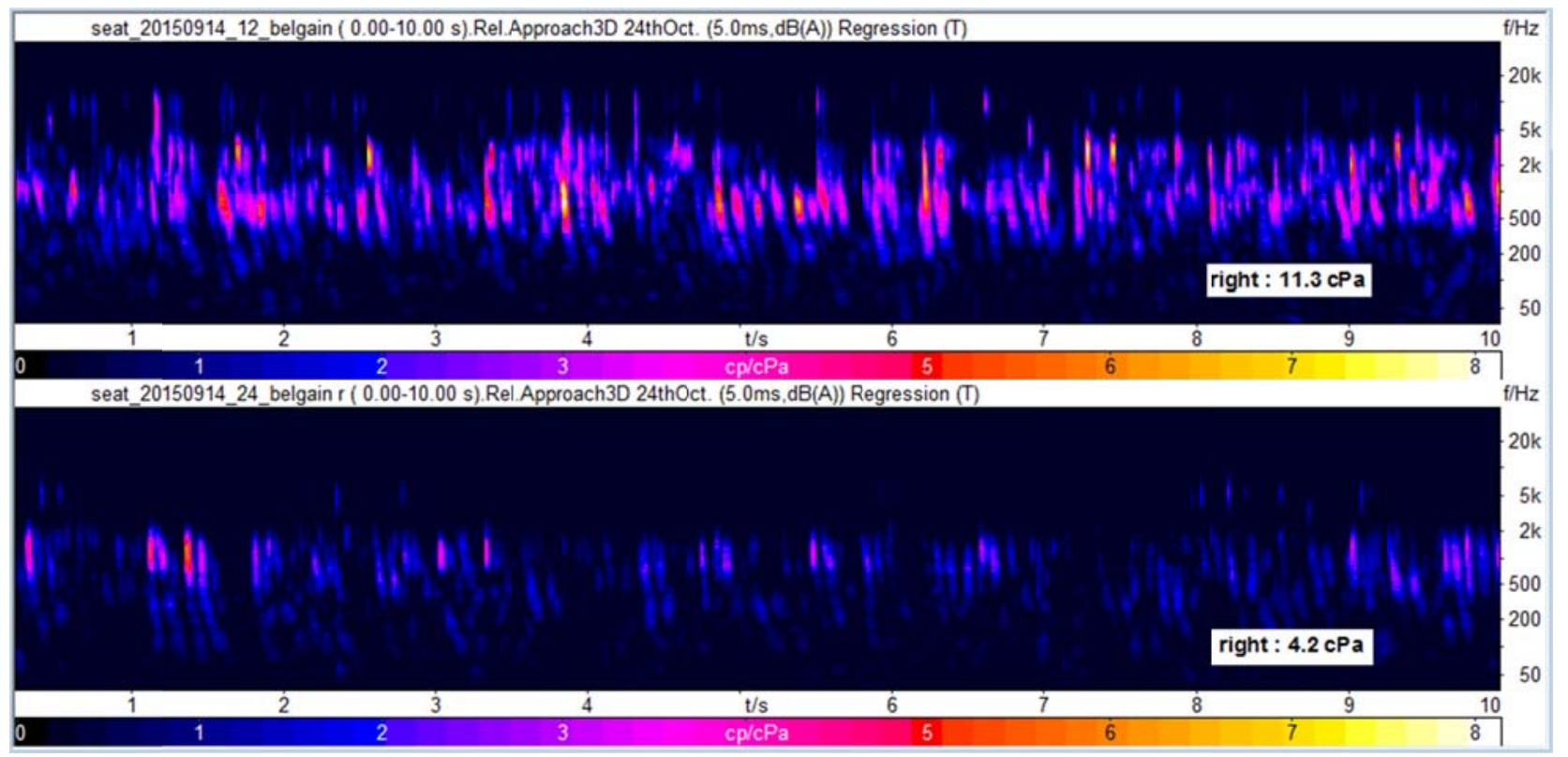

Figure 9. Relative Approach3D (Upper: With Headrest; Lower: Without Headrest)

Figure 9 indicates the signal changes of the condition without headrest much less than the condition with headrest. This analysis also focuses on sensitive frequencies of human hearing. For right ear position, Relative Approach3D value is changed from $11.3 \mathrm{cPa}$ to $4.2 \mathrm{cPa}$ when headrest 
was removed.

Based on sound and vibration measurement in hemi-anechoic lab environment, three different analytical techniques were used to identify the rattle noise source and quantify the rattle noise. Wavelet Transform analysis of both SPL and acceleration pinpoints to the joint of the seat back assembly and headrest assembly. The high correlation between SPL and acceleration level further confirmed again verified the conclusion.

Based on findings of the rattle noise source, solving the rattle noise issue is not very difficult in this case. It was found that rattle noise is caused by collision of spring bolts for headrest height adjustment and the headrest post. Figure 10 is the schematic diagram of the joint area. The collision happens since the head rest assembly was loose. Therefore, the design change of the headrest mount guide and spring bolt stiffness are implemented, which ensures headrest assembly and seatback assembly have tight contact, the rattle noise issue which customers had complaints was solved. The details of design change and further validation were not included in this paper.

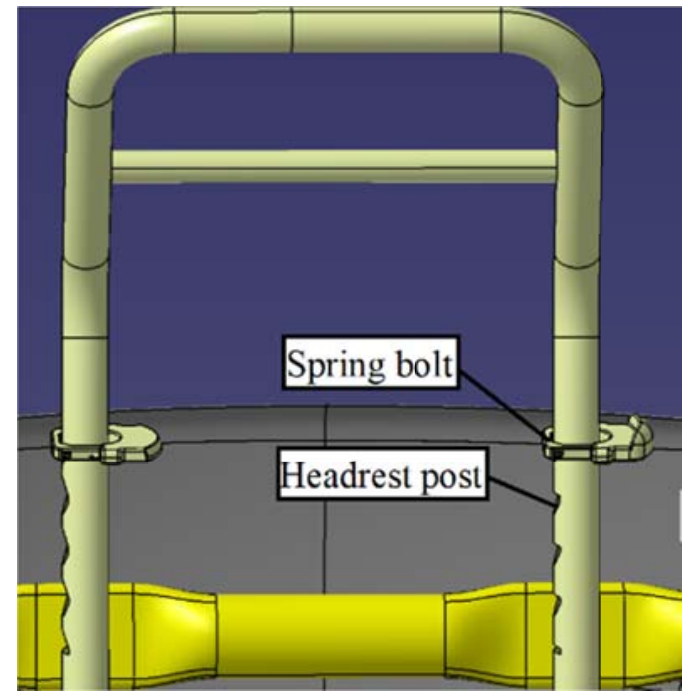

Figure 10. Schematic diagram of the joint area

\section{Conclusion}

A vehicle interior rattle noise was identified by measuring sound pressure and vibration around suspected component, which is the passenger side seat. Multiple signal analysis techniques were used in this case for identifying, confirming the rattle noise source and qualifying the rattle noise. Wavelet Transform analysis technique was selected since it has capability of capturing the transient phenomenon of abnormal noise. Both sound pressure and vibration Wavelet Transform analysis indicate that the joint of headrest assembly and seat back is the source of rattle noise. Relative Appaoch3D technology was also used to conduct quantitative analysis of the rattle noise.

The study case shows that after on-road subjective evaluation, reconstructing rattle noise source from suspected component in hemi anechoic lab environment, measuring both sound and vibration signal, utilizing multiple signal analysis techniques proved to be effective way to identifying, confirming and quantifying the rattle noise source.

\section{References}

[1] Farokh Kavarana, Benny Rediers, Squeak and Rattle-State of the Art and Beyond, J., SAE, 1999-01-1728.

[2] Fang Chen, Martin Trapp, Automotive Buzz, Squeak and Rattle, first edition, United States of 
America, 2012.

[3] Frusti, Fletcher, Grinn, Gu, Vehicle Seat Sub-System Squeak and Rattle Testing and Requirements, IMAC-XIV, SEM, pp. 497-501, 1996.

[4] S. A. Nolan, Yao, Tran, Weber, Heard, Instrument Panel Squeak and Rattle Testing and Requirements, IMAC-XIV, SEM, pp. 490-494, 1996.

[5] Su-Hyun Shin, Cheolung Cheong, Experimental Characterization of Instrument Panel Buzz, Squeak and Rattle (BSR) in a Vehicle, J., Applied Acoustics 71 (2010) 1162-1168.

[6] R. P. Senthil Kumar, N. Jaya Kumar, Sajith Nair, Seat Squeak Measurement and Diagnosis, J., SAE International, 2013-26-0094. 